\title{
Atrésia Esofágica: Um Desafio desde o Diagnóstico Pré-natal ao Tratamento Cirúrgico
}

\section{Esophageal Atresia: A Challenge from Prenatal Diagnosis to Surgical Treatment}

Vera Trocado1,2,3 , Catarina Barroso 2,3,4 , Cristina Nogueira-Silva 2,3,5, Jorge Correia-Pinto $2,3,4$

\section{RESUMO}

A atrésia esofágica é a anomalia congénita mais comum do desenvolvimento do esófago, caracterizando-se por uma disrupção da sua continuidade, com uma prevalência de cerca de 1 em cada 2500 a 4500 nados vivos. A abordagem desta condição, desde o seu diagnóstico no período pré-natal até ao tratamento cirúrgico no período neonatal, constitui um importante desafio, tanto para o obstetra como para o cirurgião pediátrico. No âmbito do diagnóstico pré-natal, a ecografia, a ressonância magnética, bem como a análise bioquímica do líquido amniótico poderão ser úteis. Por outro lado, no que diz respeito ao tratamento cirúrgico desta condição, dispomos hoje de técnicas cirúrgicas minimamente invasivas, associadas a menor número de sequelas musculoesqueléticas, menor dor no pós-operatório e a um melhor resultado estético final. Neste artigo, os autores fazem uma revisão do papel das diferentes técnicas de diagnóstico pré-natal disponíveis, bem como das diferentes abordagens para o tratamento cirúrgico.

PALAVRAS-CHAVE: Atrésia Esofágica; Fístula Traqueoesofágica; Diagnóstico Pré-natal; Toracoscopia

\section{ABSTRACT}

Esophageal atresia is the most common congenital anomaly of the esophagus, characterized by disruption of its continuity, with a prevalence of 1 per 2500 to 4500 live births.

The approach to this condition, since diagnosis in the prenatal period to surgical treatment in the neonatal period, is an important challenge, both for the obstetrician and the pediatric surgeon. Regarding to prenatal diagnosis, ultrasound, magnetic resonance, and biochemical analysis of amniotic fluid are relevant. On the other hand, concerning to surgical treatment, nowadays there are available minimal invasive surgical techniques, with less musculoskeletal sequelae, less pain in the post-operative period and a better esthetic final result. In this article, the authors review the different prenatal diagnostic techniques available, as well as different approaches to the surgical treatment.

KEYWORDS: Esophageal Atresia; Prenatal Diagnosis; Tracheoesophageal Fistula; Thoracoscopy

1. Serviço de Ginecologia e Obstetrícia, Unidade Local de Saúde do Alto Minho, Viana do Castelo, Portugal. 2. Instituto de Investigação em Ciências da Vida e da Saúde, Escola de Ciências da Saúde, Universidade do Minho, Braga, Portugal. 3. Instituto de Investigação em Ciências da Vida e da Saúde/3B's - Laboratório Associado do Governo Português, Braga/Guimarães, Portugal. 4. Serviço de Cirurgia Pediátrica, Hospital de Braga, Braga, Portugal. 5. Serviço de Ginecologia e Obstetrícia, Hospital de Braga, Braga, Portugal.

Recebido: 27/05/2016 - Aceite: 29/05/2016 


\section{INTRODUÇÃO}

A atrésia esofágica é a anomalia congénita mais comum do desenvolvimento do esófago, caracterizando-se por uma disrupção da sua continuidade. A sua prevalência é de cerca de 1 em cada 2500 a 4500 nados vivos e em mais de $90 \%$ dos casos associa-se a fístula traqueoesofágica. ${ }^{1}$ Segundo o sistema de classificação de Gross (Fig. 1), existem 5 tipos de anomalias, de acordo com a presença ou ausência de fístula traqueoesofágica e a sua localização. A forma mais comum, correspondente a cerca de $85 \%$ dos casos, é a atrésia esofágica com fístula traqueoesofágica distal (tipo C). Com menor frequência (7\%) pode manifestar-se como atrésia esofágica pura sem fístula associada (tipo A), com fístula traqueoesofágica proximal (2\%; tipo B), fístula proximal e distal (<1\%); tipo D) ou sob a forma de fístula em $\mathrm{H}$ sem atrésia esofágica (4\%; tipo E). ${ }^{2}$

Em cerca de 50\% dos casos, associa-se a outras anomalias genéticas, cromossómicas ou estruturais, tais como a síndrome VACTERL (anomalias vertebrais, anorretais, cardíacas, traqueais, esofágicas, renais e dos membros), a síndrome CHARGE (coloboma, anomalias do sistema nervoso central, coração, atrésia das coanas, atraso de crescimento, defeitos urinários ou genitais e distúrbios da audição) ou a trissomia 18.

A apresentação clínica caracteriza-se pela formação de bolhas de saliva na boca e nariz do recém-nascido, bem como por episódios de tosse, cianose e dificuldade respiratória, exacerbados pela alimentação, capazes de condicionar regurgitação e consequente aspiração.

Perante esta suspeita, usualmente o diagnóstico pós-natal pode ser feito através da impossibilidade de progredir uma sonda nasogástrica ou orogástrica e da confirmação radiográfica da não progressão da sonda, visualizando-se esta no topo esofágico proximal (Fig. 2). Na grande maioria dos casos, a visualização radiográfica da bolha gástrica indica a coexistência de uma fístula traqueoesofágica distal. ${ }^{3}$

\section{DIAGNÓSTICO PRÉ-NATAL}

O estabelecimento do diagnóstico de atrésia esofágica no período pré-natal, à semelhança da grande maioria das outras anomalias congénitas, é preferencial por várias razões. Em primeiro lugar, permite o aconselhamento e preparação do casal para o nascimento de um filho que necessitará de cuidados especiais. Em segundo lugar, permite o planeamento do parto e articulação da transferência do recém-nascido para uma unidade com cuidados diferenciados em cirurgia neonatal. Por último, a existência de um diagnóstico pré-natal evita a alimentação precoce de um recém-nascido afetado, reduzindo assim o número de casos de pneumonia de aspiração. Poder-se-á, desta forma, inferir que um diagnóstico pré-natal terá um impacto positivo nos outcomes pós-natais. Ao invés, um resultado falso positivo poderá levar a ansiedade e stress desnecessários por parte das famílias.

A identificação pré-natal desta condição é complexa e constitui um importante desafio para o obstetra, ocorrendo em apenas cerca de um terço dos casos. ${ }^{4}$

\section{ECOGRAFIA OBSTÉTRICA}

O diagnóstico de atrésia esofágica, habitualmente, é suspeitado no terceiro trimestre de gravidez, com base em sinais ecográficos indiretos. A suspeita diagnóstica baseia-se na presença de hidrâmnios e/ou na ausência ou existência de bolha gástrica de pequenas dimensões. O valor preditivo destes sinais é baixo, por vários motivos. Em primeiro lugar, sabemos que a deglutição fetal é intermitente, pelo que a não visualização da bolha gástrica pode significar apenas que não ocorreram deglutições recentemente e o estômago encontra-se vazio.
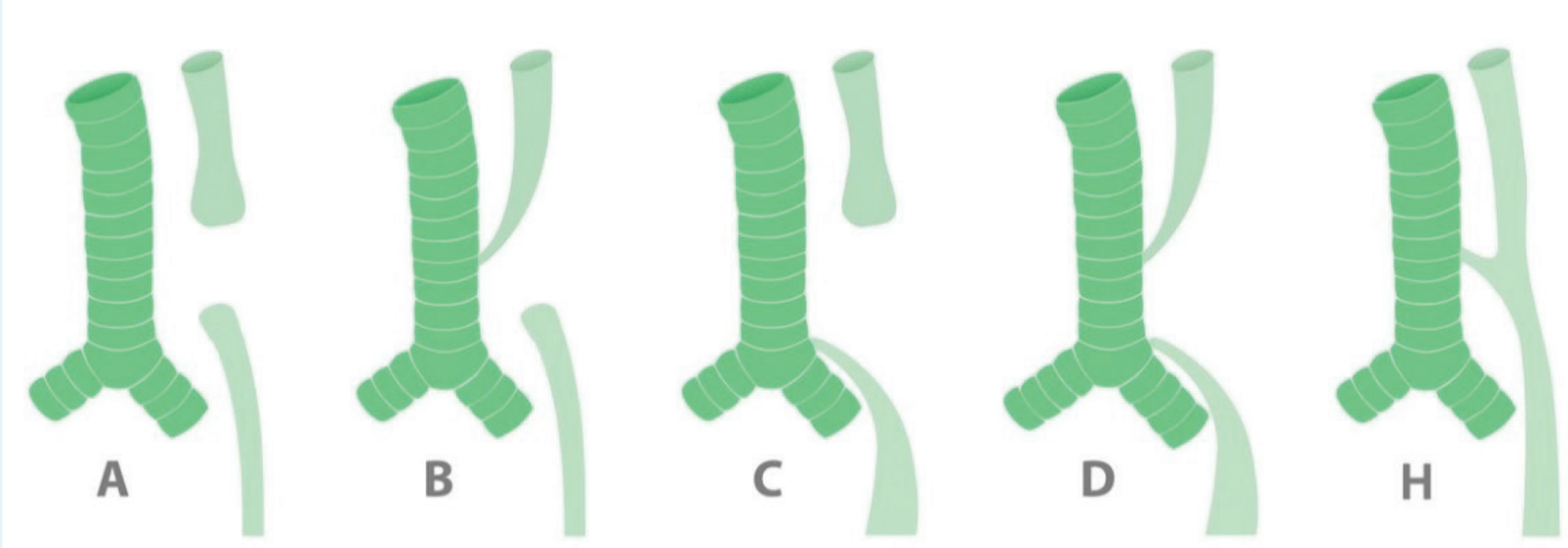

FIGURA 1. Tipos de atrésia esofágica, de acordo com a classificação de Gross. 

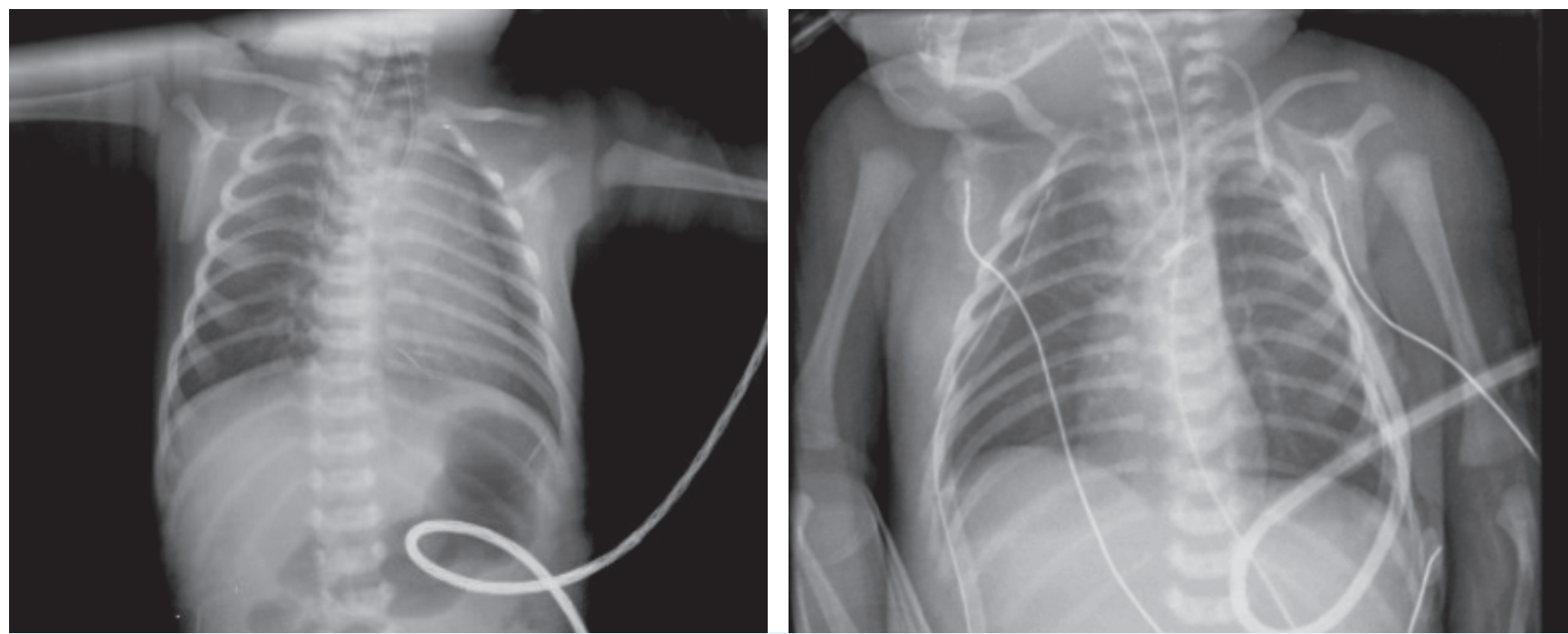

FIGURA 2. A - Ausência de progressão de sonda orogástrica em radiografia. B - Demonstração da continuidade do esófago com progressão da sonda até ao estômago, no pós-operatório.
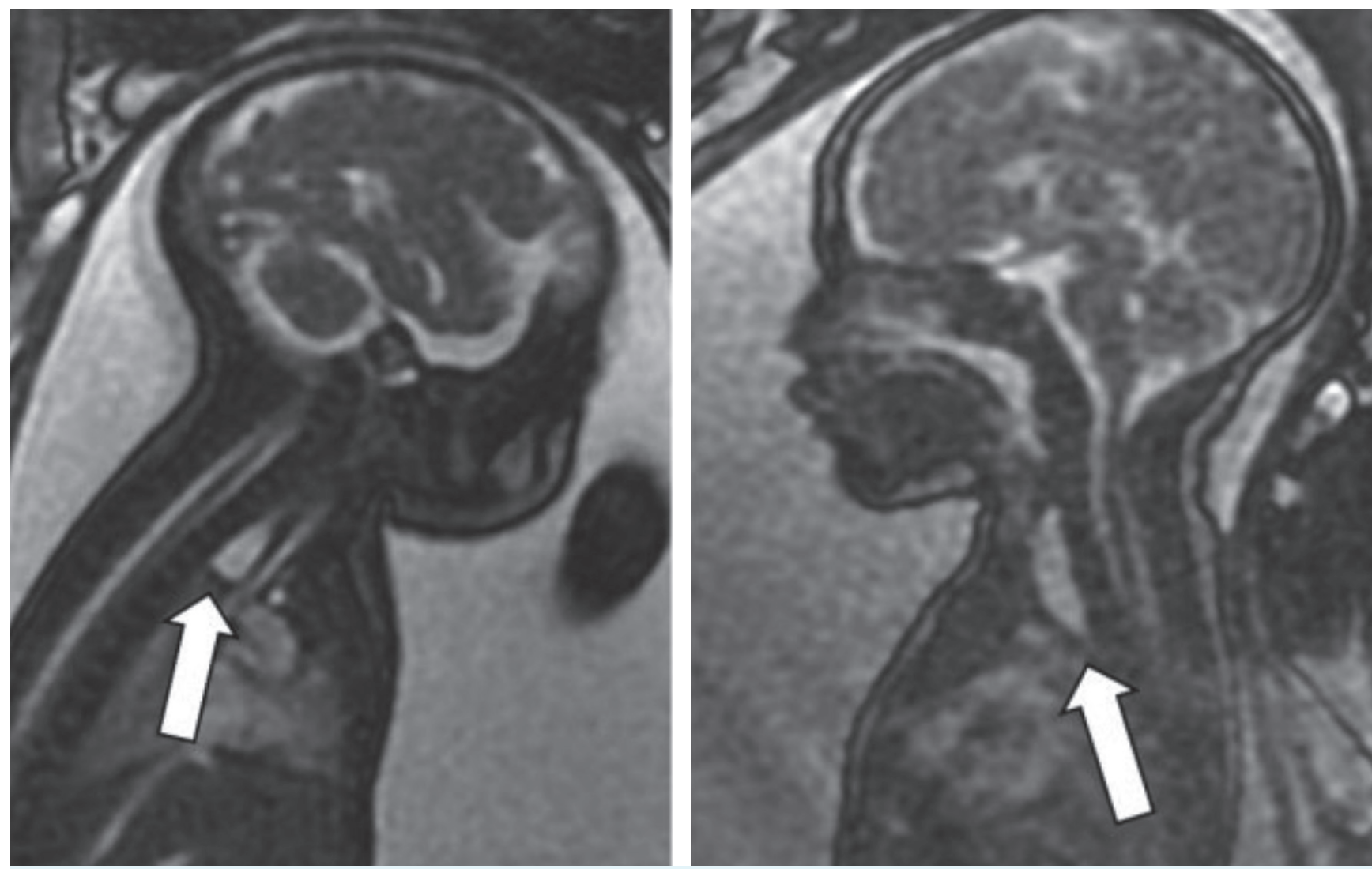

FIGURA 3. Imagem de ressonância magnética, observando-se o "pouch sign" (seta) durante a deglutição fetal. Com permissão, Salomon LJ, et al. Ultrasound Obstet Gynecol. Berlin: John Wiley \& Sons; 2009.

Em segundo lugar, a grande maioria dos casos de atrésia esofágica associa-se a fístula traqueoesofágica distal, pelo que poderá ser visualizada a bolha gástrica devido à passagem de líquido amniótico através da fístula. Na inexistência de fístula, o estômago poderá ser também visualizado devido ao preenchimento por secreções da mucosa gástrica. ${ }^{5}$ Por outro lado, o hidrâmnios está presente em cerca de $10 \%$ de todas as gestações, e a atrésia esofágica corresponde apenas a cerca de $1 \%$ dos casos de hidrâmnios isolado, pelo que este constitui um acha- do inespecífico. Para além disso, nem todos os casos de atrésia esofágica se associam ao desenvolvimento de hidrâmnios, sendo reportado em cerca de 58\% dos casos. Inúmeras outras causas, tais como o atingimento neuromuscular, anomalias do sistema nervoso central ou anomalias musculoesqueléticas faciais podem condicionar a existência de hidrâmnios e ausência de bolha gástrica, por atingimento da deglutição. Desta forma, a combinação destes achados resulta num valor preditivo positivo baixo, entre 40 e $56 \% .^{5-7}$ 


\section{RESSONÂNCIA MAGNÉTICA FETAL}

Naúltima década, a progressiva experiência na aplicação da ressonância magnética fetal no diagnóstico pré-natal, tornou esta técnica hoje disponível como método de segunda linha, nos casos em que é colocada a suspeita ecográfica. Langer et al definiram como critério de diagnóstico, numa ressonância magnética $T 2$, a não visualização da porção intratorácica do esófago, com uma sensibilidade de $100 \%$, especificidade de $80 \%$ e valor preditivo positivo de $83 \% .{ }^{8}$ A maior facilidade na localização do plano médio-sagital e na visualização do "pouch sign" parecem ser importantes vantagens da ressonância magnética, com impacto positivo nas taxas de deteção (Fig. 3). No entanto, encontra-se ainda limitada a determinados centros, não constituindo na atualidade uma técnica custo-efetiva como método de rastreio. ${ }^{6}$

\section{ANÁLISE BIOQUÍMICA DO LÍQUIDO AMNIÓTICO}

Mais recentemente, Czerkiewics et al descreveram a análise bioquímica do líquido amniótico como método aplicável aos casos em que há suspeita ecográfica de atrésia esofágica. A existência de um padrão de enzimas digestivas no líquido amniótico característico desta condição permitiu a criação de um índice (alfa-fetoproteína (MoM) x gamaglutamiltranspeptidase (MoM)), com um cutoff de 3, com sensibilidade de $98 \%$ e especificidade de 100\% para o diagnóstico. No entanto, esta avaliação foi realizada apenas em casos em que existia hidrâmnios, pelo que não é conhecido o papel da avaliação bioquímica do líquido amniótico nos casos em que o volume de líquido é normal.?

Apesar dos avanços tecnológicos do último século e da consequente evolução da medicina fetal e neonatal, temos ainda um longo caminho a percorrer no diagnóstico pré-natal desta condição. A avaliação ecográfica por um operador experiente é determinante na identificação dos casos suspeitos. A evidência mais recente aponta para que nestes casos deva ser proposta a realização de uma ressonância magnética entre as 30-32 semanas. A análise bioquímica do líquido amniótico pode ser útil, merecendo ainda particular atenção em estudos de maior escala. ${ }^{10}$

\section{TRATAMENTO CIRÚRGICO}

A abordagem cirúrgica para a correção da atrésia do esófago depende das características da malformação, nomeadamente da existência ou não de fístula traqueo- esofágica (proximal e/ou distal) e a distância entre os topos esofágicos.

A cirurgia de correção está contraindicada nos casos de patologia concomitante com prognóstico reservado, como a trissomia 18, síndrome de Potter e malformações cardíacas graves, incompatíveis com a vida.

\section{ATRÉSIA DO ESÓFAGO DE TIPO CE D}

A atrésia do esófago de tipo C é aquela em que há maior consenso sobre o algoritmo de tratamento a ser utilizado. Após o seu diagnóstico, a cirurgia imediata raramente é necessária e deve, idealmente, ser agendada para 12 a 24 horas depois da admissão do recém-nascido. Na maioria dos casos é possível fazer a laqueação da fístula traqueoesofágica e a correção da atrésia esofágica com anastomose dos dois topos do esófago num procedimento único. A anastomose primária deve ser tentada mesmo que fique sob tensão extrema.

Durante muitos anos, a atrésia do esófago com fístula traqueoesofágica distal foi corrigida com sucesso por toracotomia. Durante o seguimento das crianças submetidas a toracotomia, um número significativo de sequelas musculoesqueléticas foi reportado, como a escápula alada, escoliose e assimetria da parede torácica por atrofia do músculo serreado anterior. A introdução da toracoscopia possibilitou um acesso à cavidade torácica menos traumático, ao qual se associam menos sequelas musculoesqueléticas, menos dor no pós-operatório, menor duração da ventilação mecânica e um melhor resultado estético (Fig. 4). Para o cirurgião, a toracoscopia permite uma visualização privilegiada da anatomia da cavidade torácica, providenciando, entre outros, precisão na identificação e laqueação da fístula no ponto onde se insere na traqueia. Apresenta uma curva de aprendizagem exigente, que requer motivação e horas de treino, mas é considerado um procedimento seguro, com resultados pós-operatórios comparáveis à técnica clássica, e elimina os efeitos musculoesqueléticos indesejáveis que durante anos foram associados à toracotomia. ${ }^{11}$

Assim, apesar do procedimento clássico ser ainda utilizado, o tratamento toracoscópico tornou-se o procedimento standard em muitos centros de Cirurgia Pediátrica em todo o mundo. ${ }^{12}$ Desde que a equipa cirúrgica e anestésica tenha experiência nesta abordagem, quase todos os recém-nascidos são candidatos ao tratamento toracoscópico. As contraindicações relativas são a prematuridade extrema, microssomia, instabilidade hemodinâmica ou respiratória. 


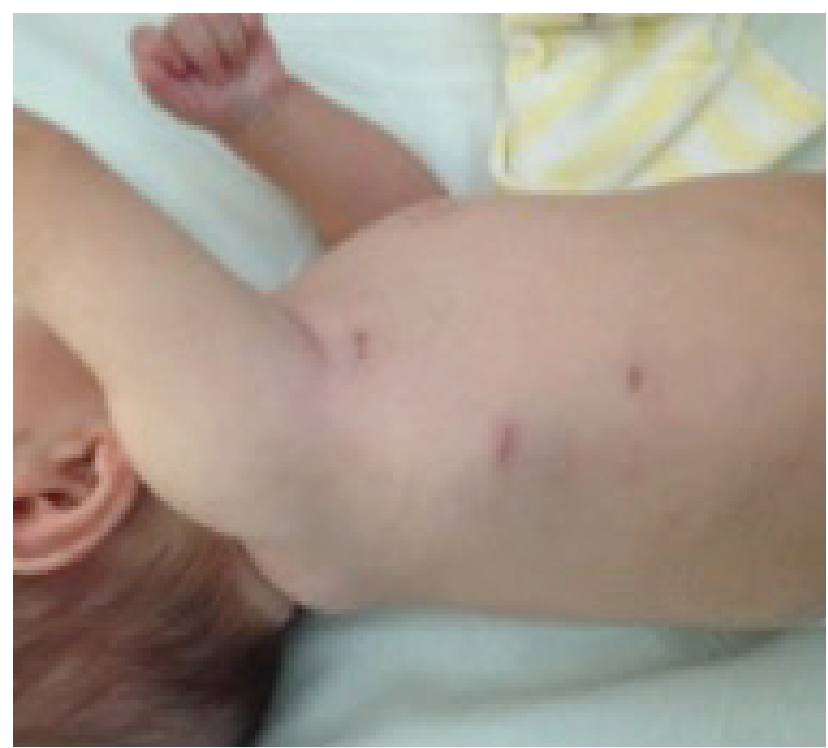

FIGURA 4. Aspeto final das cicatrizes cirúrgicas, por abordagem toracoscópica.

Idealmente, a broncoscopia antes da cirurgia permite a identificação exata da localização da fístula traqueoesofágica, facilitando a abordagem da via aérea pela equipa anestésica e o planeamento do procedimento cirúrgico. É ainda possível identificar a presença de fístula traqueoesofágica proximal associada, na eventualidade de se tratar de uma atrésia do esófago de tipo D, cujo diagnóstico clínico-imagiológico é difícil e pode até passar despercebido durante o procedimento cirúrgico. Depois da colocação de um tubo endotraqueal, sob anestesia geral, o recém-nascido é colocado na posição de Cushiery (quase em decúbito ventral, com o lado direito elevado a $30^{\circ}$ ), para otimizar o acesso ao mediastino posterior. A abordagem é realizada pelo hemitórax direito. $\bigcirc$ cirurgião principal e ajudante posicionam-se à frente do recém-nascido, enquanto o monitor é colocado atrás. $O$ procedimento pode ser realizado com 3 portas cirúrgicas, mas ocasionalmente são necessárias 4 . 0 pneumotórax com dióxido de carbono é utilizado para colapsar o pulmão. A dissecção da pleura do mediastino posterior distalmente à veia ázigos, permite o acesso à fístula traqueoesofágica que é dissecada. Cranialmente à veia ázigos, a porção proximal do esófago é isolada da traqueia, sendo depois seccionada a sua extremidade. A fístula é laqueada com 2 clips e seccionada distalmente. A anastomose das duas porções do esófago é realizada com suturas separadas, em uma camada única. A sonda transanastomótica utilizada para a finalização da anastomose mantém-se no final da cirurgia e é colocado um dreno torácico. ${ }^{11}$

A abordagem da atrésia do esófago de tipo Dé semelhante à anteriormente descrita. Não raras vezes, o diagnóstico é feito intraoperatoriamente, exigindo a laqueação e secção das duas fístulas no mesmo tempo operatório.

\section{ATRÉSIA DO ESÓFAGO DE TIPO A E B}

As atrésias esofágicas de tipo A e B, sem fístula distal, têm maior probabilidade de ser long gap (os topos proximal e distal encontram-se muito distantes um do outro).

Enquanto que na atrésia do esófago de tipo C é consensual que a correção deve ser realizada nos primeiros dias de vida e deve sempre ser tentada a anastomose primária, nas atrésias do esófago long gap há várias abordagens alternativas e a experiência de cada cirurgião determina a técnica a ser utilizada. ${ }^{13}$

Do ponto de vista cirúrgico, a correção da atrésia do esófago long gap permanece um desafio. Independentemente da técnica utilizada, está associada a complicações no período pós-operatório e a morbilidade considerável. As técnicas utilizadas incluem procedimentos com preservação do esófago, técnicas de alongamento e de substituição do esófago.

Apesar da introdução de inúmeras novas técnicas ao longo dos anos, muitos cirurgiões acreditam que a anastomose primária é a melhor abordagem. Optam por uma atitude inicial conservadora e protelam a anastomose até que o crescimento natural aproxime os dois topos esofágicos, desde que o recém-nascido se mantenha estável, o que pode ser dificultado pela presença de uma fístula proximal. Durante este período deve manter-se uma sonda em aspiração contínua do topo proximal. Muitos cirurgiões pediátricos optam por fazer a gastrostomia como procedimento inicial, embora seja possível manter o recém-nascido com nutrição parentérica durante algumas semanas até à cirurgia de correção.

Na técnica de Foker os dois topos esofágicos são aproximados por tração externa durante determinado período de tempo, para a posterior realização da anastomose primária. A taxa de sucesso é considerável e os resultados a longo prazo são satisfatórios, mas à custa de uma taxa de morbilidade significativa. ${ }^{14,15}$

Foram também reportadas técnicas que utilizam compressão magnética para aproximação dos topos esofágicos, até à sua união e anastomose. Não raras as complicações, esta provou ser uma técnica viável em casos selecionados. ${ }^{16,17}$

As técnicas de substituição esofágica são consideradas por alguns a melhor solução quando a distância entre os topos impossibilita, ou há falência, da anastomose primária. As mais utilizadas são a interposição do cólon, transposição gástrica, transposição do tubo gástrico e interposição do jejuno. Não são reportadas diferenças significativas entre os resultados obtidos. Apesar de se- 
rem viáveis, todas estão associadas a grande morbilidade, pelo que não há consenso sobre qual o melhor método de substituição. ${ }^{18}$

\section{ATRÉSIA DO ESÓFAGO DO TIPO H}

Estes recém-nascidos beneficiam da broncoscopia pré- operatória para localização e canalização da fístula para otimizar o acesso durante a cirurgia. O procedimento consiste na laqueação e secção da fístula e pode ser realizado por toracotomia, cervicotomia ou toracoscopia. ${ }^{19}$

\section{CONCLUSÃO}

A taxa de sobrevivência de recém-nascidos com atrésia esofágica é atualmente superior a 90\%, embora a prevalência de complicações a longo prazo, como a disfagia, o refluxo gastroesofágico, as infeções respiratórias de repetição ou a tosse persistente, seja ainda comum. ${ }^{20,21}$

Apesar do importante progresso no tratamento cirúrgico que decorreu nos últimos anos, são necessários esforços no sentido de otimizar o diagnóstico pré-natal desta condição, bem como na otimização do follow-up a longo prazo destas crianças.

CONFLITOS DE INTERESSE: Os autores declaram a inexistência de conflitos de interesse na realização do trabalho.

FONTES DE FINANCIAMENTO: Não existiram fontes externas de financiamento para a realização deste artigo.

\section{REFERÊNCIAS}

1. Cassina M, Ruol M, Pertile R, Midrio P, Piffer S, Vicenzi V, et al. Prevalence, characteristics, and survival of children with esophageal atresia: A 32-year population-based study including 1,417,724 consecutive newborns. Birth Defects Res Part A Clin Mol Teratol. 2016;106:542-8.

2. Rothenberg SS. Esophageal atresia and tracheoesophageal fistula malformations. 6th ed. Amsterdam: Elsevier; 2014.

3. Atresia E. Congenital anomalies. 20th ed. Amsterdam: Elsevier; 2016.

4. Spaggiari E, Faure G, Rousseau V, Sonigo P, Millischer-Bellaiche AE, Kermorvant-Duchemin E, et al. Performance of prenatal diagnosis in esophageal atresia. Prenat Diagn. 2015;35:888-93.

5. Houfflin-Debarge $V$, Bigot J. Ultrasound and MRI prenatal diagnosis of esophageal atresia: effect on management. J Pediatr Gastroenterol Nutr. 2011;52:S9-S11.
6. Bradshaw CJ, Thakkar H, Knutzen L, Marsh R, Pacilli M, Impey $L$, et al. Accuracy of prenatal detection of tracheoesophageal fistula and oesophageal atresia. J Pediatr Surg. 2016:1-5.

7. Choudhry M, Boyd PA, Chamberlain PF, Lakhoo K. Prenatal diagnosis of tracheo-oesophageal fistula and oesophageal atresia. Prenat Diagn. 2007;27:608-10.

8. Langer JC, Hussain H, Khan A, Minkes RK, Gray D, Siegel $\mathrm{M}$, et al. Prenatal diagnosis of esophageal atresia using sonography and magnetic resonance imaging. J Pediatr Surg. 2001;36:804-7.

9. Czerkiewicz I, Dreux S, Beckmezian A, Benachi A, Salomon LJ, Schmitz T, et al. Biochemical amniotic fluid pattern for prenatal diagnosis of esophageal atresia. Pediatr Res. 2011;70:199202.

10. Garabedian C, Verpillat P, Czerkiewicz I, Langlois C, Muller F, Avni F, et al. Does a combination of ultrasound, MRI, and biochemical amniotic fluid analysis improve prenatal diagnosis of esophageal atresia? Prenat Diagn. 2014;34:839-42.

11. Holcomb GW 3rd, Rothenberg SS, Bax KM, Martinez-Ferro M, Albanese CT, Ostlie DJ, et al. Thoracoscopic repair of esophageal atresia and tracheoesophageal fistula: a multi-institutional analysis. Ann Surg. 2005;242:422-30.

12. Borruto F, Impellizzeri P, Montalto A, Antonuccio P, Santacaterina E, Scalfari G, et al. Thoracoscopy versus thoracotomy for esophageal atresia and tracheoesophageal fistula repair: Review of the literature and meta-analysis. Eur J Pediatr Surg. 2012;22:415-9.

13. Rothenberg SS. Thoracoscopic repair of esophageal atresia and tracheo-esophageal fistula in neonates: evolution of a technique. J Laparoendosc Adv Surg Tech. 2012;22:195-9.

14. Foker JE, Linden BC, Boyle EM, Marquardt C. Development of a true primary repair for the full spectrum of esophageal atresia. Ann Surg. 1997;226:533-541; discussion 541-543.

15. Bobanga ID, Barksdale EM. Foker Technique for the management of pure esophageal atresia: long-term outcomes at a single institution. Eur J Pediatr Surg. 2016;26:215-8.

16. Zaritzky M, Ben R, Johnston K. Magnetic gastrointestinal anastomosis in pediatric patients. J Pediatr Surg. 2014;49:1131-7.

17. Dorman RM, Vali K, Harmon CM, Zaritzky M, Bass KD. Repair of esophageal atresia with proximal fistula using endoscopic magnetic compression anastomosis (magnamosis) after staged lengthening. Pediatr Surg Int. 2016;32:525-8.

18. Loukogeorgakis SP, Pierro A. Replacement surgery for esophageal atresia. Eur J Pediatr Surg. 2013;23:182-90.

19. Al-Salem AH, Mohaidly M Al, Al-Buainain HM, Al-jadaan S, Raboei E. Congenital H-type tracheoesophageal fistula: a national multicenter study. Pediatr Surg Int. 2016;32:487-91.

20. Connor MJ, Springford LR, Kapetanakis WV, Giuliani S. Esophageal atresia and transitional care - Step 1: A systematic review and meta-analysis of the literature to define the prevalence of chronic long-term problems. Am J Surg. 2015;209:747-59.

21. Dellenmark-Blom M, Chaplin JE, Gatzinsky V, Jönsson L, Wigert $\mathrm{H}$, Apell J, et al. Health-related quality of life experiences among children and adolescents born with esophageal atresia: Development of a condition-specific questionnaire for pediatric patients. J Pediatr Surg. 2016;51:563-9. 